\title{
Solutions to Penicillium taxonomy crucial to mycotoxin research and health
}

\author{
R. Russell M. Paterson, Armando Venâncio, Nelson Lima* \\ Micoteca da Universidade do Minho, Centro de Engenharia Biológica, Campus de Gualtar, 4710-057 Braga, Portugal \\ Received 18 March 2004; accepted 2 April 2004 \\ Available online 10 May 2004
}

\begin{abstract}
The stability of taxonomy within Penicillium is reviewed with respect to mycotoxin production. Identification remains difficult despite the availability of modern methods. Proficiency testing is rare and conventional identifications do not inform reliably as to whether mycotoxins were detected/produced. A solution which consists of identifying a Penicillium strain as terverticillate and then undertaking mycotoxin analysis is described.

(c) 2004 Elsevier SAS. All rights reserved.
\end{abstract}

Keywords: Terverticillate; Penicillia; Ochratoxin A; Patulin; Taxonomy; Monophyletic

\section{Introduction}

"Men, women and children died in unbearable agonising pain." (Tenth century chronicler of "St. Anthony's Fire" caused by ergot alkaloid contaminated grain from Claviceps purpurea).

The milestone in mycotoxicology was reached when 100000 turkeys died in England from aflatoxin poisoning of feed. Patulin was headlined in the "popular" English press when apple juice was heralded as containing cancerous agents. Mycotoxins have reportedly been developed as biological weapons, although penicillia are not the prime targets. Though of international importance, mycotoxins are generally poorly understood and ignored by the general public.

Penicillia are microorganisms of great interest and penicillin is the most significant and well known product of the genus. Certain taxa biodeteriorate commodities (Fig. 1) and some cause rot, e.g., Penicillium expansum of apples. The organoleptic properties of food and drink are affected detrimentally by Penicillium growth. They are involved in recycling of organic matter in the environment, while production of cheeses (e.g., Camembert and Roquefort) relies on mem-

\footnotetext{
* Corresponding author.

E-mail address: nelson@iec.uminho.pt (N. Lima).
}

bers of this genus. Organic acids and enzymes are produced, but industrial exploitation is hampered by toxic secondary metabolite production.

Mycotoxins are frequently present in food and feedstuff and are associated with illness from damp homes. The economies of certain countries are harmed by their occurrence and need for control. Developing countries may be severely affected because of optimal climates needed for production and poor commodity growth and storage conditions. Better quality material is exported, leaving poorer grade material for local populations.

The total number of mycotoxins is unknown. Toxic fungal metabolites may be in the thousands. Approximately 6 are considered to be of major importance. Patulin and ochratoxin A (OTA) (Fig. 2) are the most significant of the Penicillium metabolites and EU statutory limits have been imposed in some foods. There are approximately 10 at the second tier of relevance. However, any of these can assume significance if present at high enough concentrations in food. P. roqueforti is used for blue cheese production and certain strains can produce patulin [23]. This may not be problematic, as patulin is degraded by sulphur-containing proteins in milk. It is metabolised during fermentation by Saccharomyces cerevisiae, and therefore is not considered to be a problem in alcoholic cider which is not supplemented with apple juice. It is unclear as to what may occur in continuous culture. Finally, fungal antibiotics are effectively 


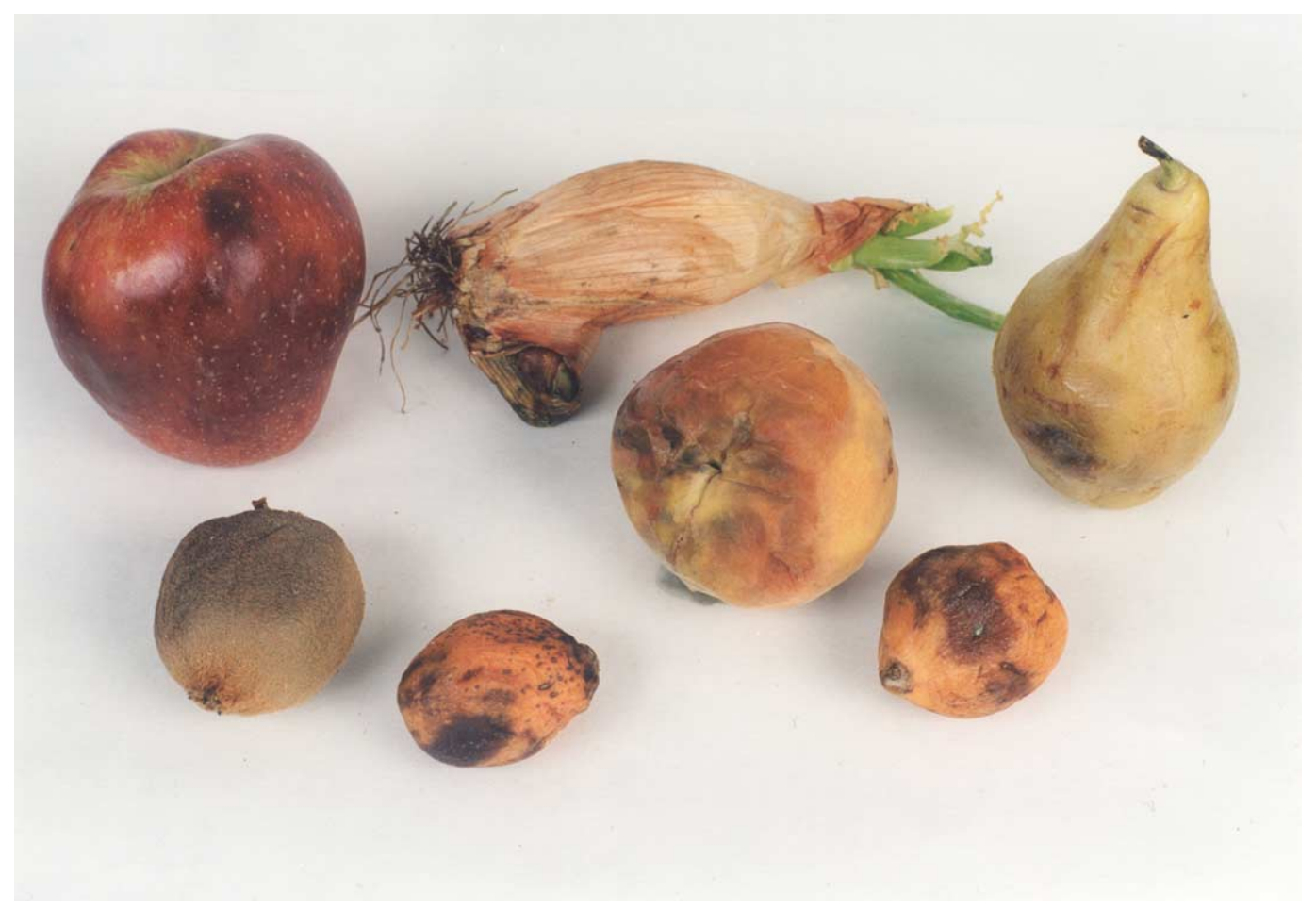

Fig. 1. Examples of fruits and vegetables susceptible to Penicillium.<smiles>O=C1C=C2C(=CCOC2O)O1</smiles>

\section{Patulin}<smiles>CC1Cc2c(Cl)cc(C(=O)NC(Cc3ccccc3)C(=O)O)c(O)c2C(=O)O1</smiles>

\section{Ochratoxin A}

Fig. 2. Chemical structures of patulin and ochratoxin A.

mycotoxins if present in non-therapeutic situations because of sensitisation and resistance effects.

The field of mycotoxins is complex and interdisciplinary, involving 5 interrelated systems [4]: (a) the commodity system consists of all aspects of production, marketing, and utilisation; (b) the spoilage system has many interrelated factors, e.g., biological, chemical, physical, microand macroenvironmental; (c) the mycotoxin system involves 5 subsystems - productivity, metabolism, toxicology, health and wealth; (d) the control system comprises prevention, mycotoxin analysis and segregation of contaminated material, and detoxification. A hazard analysis and critical control points (HACCP) approach is considered optimal; and (e) the socio-economic system can be described as cultural, political and economic.

\section{Health}

Patulin and OTA are regulated by the EU. OTA is classified as "possibly carcinogenic to humans" (group 2B) by the International Agency for Research in Cancer. Balkan endemic nephropathy, a fatal renal disease of humans, has been associated with OTA. It causes renal toxicity, nephropathy, immunosuppression and cancer in animals. OTA inhibits protein, RNA and DNA synthesis. Patulin is classified as being of "no evidence in humans" (group 3) in terms of carcinogenicity. It has mutagenic properties and is strongly antibiotic, including against Mycobacterium tuberculosis. It is immunosuppressive, and produces ulceration, congestion, and haemorrhagic lesions. The toxicity of patulin is related to enzyme alkylation, and inhibition of DNA translation/transcription. The co-occurrence of mycotoxins can enhance toxicity, and non-toxic fungal metabolites can act synergistically with toxic ones.

\section{Significance of taxonomy}

The roles of Penicillium identification in mycotoxicology are illustrated in Fig. 3. The (a) pest and disease, (b) biode- 


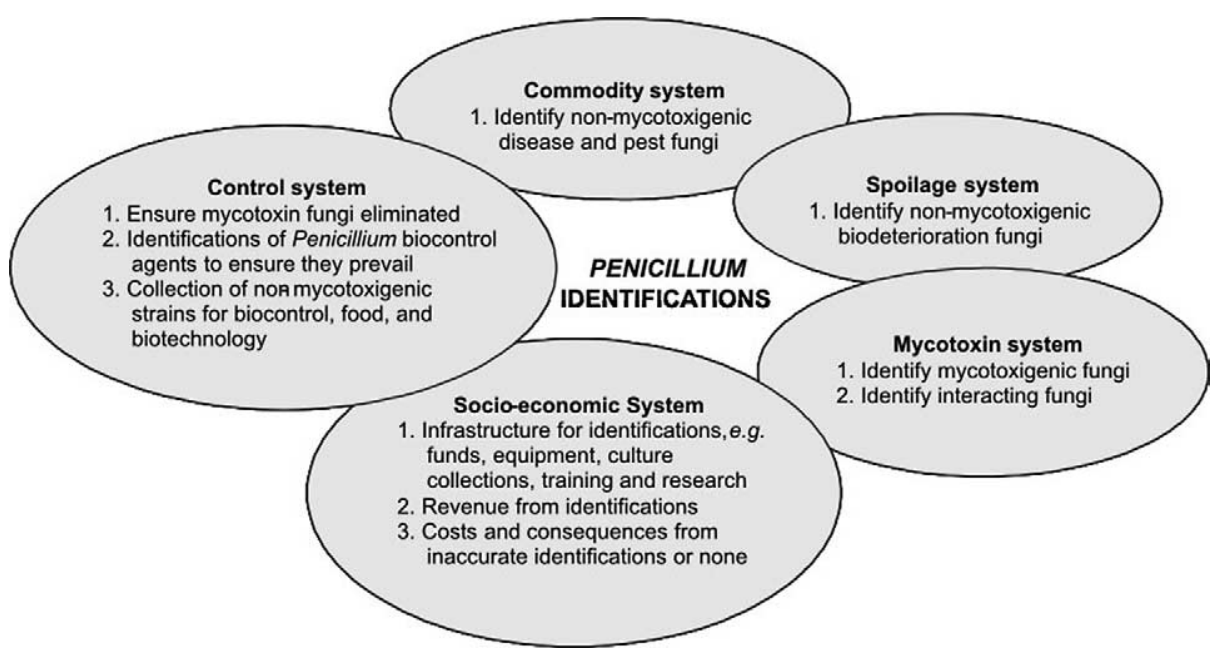

Fig. 3. The role of Penicillium identification in the commodity system. The figure is relevant to mycotoxigenic fungal identifications in general if the term "mycotoxigenic fungi" is substituted for "Penicillium".

terioration, and (c) mycotoxigenic fungi must be identified within the (a) commodity, (b) spoilage, and (c) mycotoxin systems respectively. This applies to mycotoxigenic fungal identifications in general. Interacting and harmless fungi ideally require identification.

Identifications of mycotoxigenic fungi are at best diagnostic for mycotoxin contamination in commodities. Obviously, they cannot provide information on what fungi were present previously, but may forecast mycotoxin contamination. Analyses for toxins indicate which fungi are and were present: a series of analyses can be extrapolated to predict future trends. Identifications from fomites generally are required.

Problem fungi need to be identified at acceptably low levels in the control system. Biocontrol fungi require being in place to work: they have to be identified. In addition, they should produce only acceptably low or no levels of potentially thousands of mycotoxins. Finally, the identity of low/non-mycotoxin-producing fungi for biocontrol, food (e.g., blue cheese) and biotechnology (e.g., organic acids) may be required. The socio-economic system involves providing the infrastructure for identifications, which requires increased investment. On the other hand, revenue is made from undertaking identifications. The consequences and costs of inaccurate or no identification need to be considered, e.g., if a toxin-producing strain is identified as being non-toxigenic. $P$. expansum can claim representation in each system: (a) fruit disease, (b) spoilage, (c) patulin production, and (d) control [12].

\section{The taxonomic problem}

A frequently cited review on the problems of Penicillium [17] anticipated results of a multidisciplinary study to address taxonomic difficulties [3,20]. Problems stem from reliance on subtle differences in terverticillate penicillia coni-
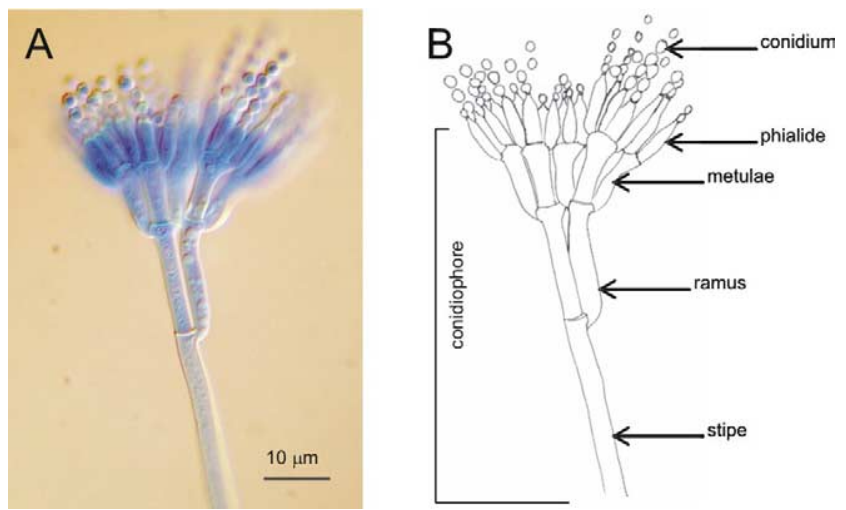

Fig. 4. Penicillium brevicompactum. (A) Differential interference contrast micrograph of a mature conidiophore stained with cotton blue. (B) Line drawing and preferred terms to describe the different structures in terverticillate penicillia.

diophores (Fig. 4). Twenty years after that study, with much water under the bridge, what is the situation? Have the results and methodology of modern methods been used widely (e.g. $[7,16,25])$ ? The answer is no. Do most scientists rely on traditional morphology? Probably yes. Identifications are still done by morphology, with a few recent physiological and chemical/molecular methods, despite the fact that many of the non-physiological and chemical features are regarded as subjective. Species level identifications are often very difficult and many errors exist in the literature. Nevertheless, research teams usually identify the isolated fungi by traditional methods and then draw important conclusions as to their significance. The Systematics in Penicillium and Aspergillus Group has produced three important books (see [25]) which are required reading for understanding the issues.

Many problems remain concerning the use of (a) various schemes for names, (b) strain variation, and (c) decisions based on minutia [17]. New varieties and species have been created only to be reclassified as members of existing taxa [10]. Novel techniques have revealed characters which were previously unreported in some species [23]. rDNA se- 
quence analyses demonstrated that the subgenus Penicillium is predominately monophyletic, and current species may be varieties [25]. Related to these, patulin has recently had statutory limits placed on its concentrations in fruit products by the EU, yet the producing species are not well defined.

A reasonable expectation is that taxonomy can predict a property. This is not always the case. Aspergillus niger was "generally recognised as safe" and a stable entity, and so is used in commercial production of organic acids and enzymes. However, it is heterogeneous and some strains produce OTA [1,32]. It is likely that a fungus from which a mycotoxin was not detected during its taxonomic designation still has that capability. Strains may not produce OTA above detection limits pre-production run but might do so during manufacturing. This suggests that analyses of product and the concept of what is an acceptable concentration are required.

Terms used to describe possible results of mycotoxin analysis are: "produced" ("detected" is better), or "not detected". "Not produced" is unacceptable. It relates to the detection limits of the analytical system used. Limits of detection and theories of uncertainty for OTA are discussed in [27]. A similar approach is required in chemotaxonomy and preservation studies, etc. Appropriate genetic analyses for a metabolic pathway can avoid these problems to a large extent, although such analyses are rare [24]. Toxigenic species could not be separated from food-production species from Aspergillus section Flavi using AFLP fingerprints, in another illustration of possible problems [14].

The relationship between fungal classification and biochemistry has been established most clearly for secondary metabolites (e.g., [10]). Metabolites can identify a taxon and indicate whether it produces a secondary metabolite of interest, unlike other methods. However, many compounds can be used in these schemes, and/or the data do not necessarily indicate whether the most important mycotoxins are produced [16]. A useful contribution came from the Danish mycology group which has a web site for images of fungi and chromatograms (http://www.biocentrum.dtu.dk/mycology/).

The difficulty in identifying filamentous fungi is highly pronounced in Penicillium, leading to misidentifications in the literature [7]. There are few "proficiency tests" in fungal identification, unlike mycotoxin analysis. Ref. [11] demonstrated inconsistency between participants for terverticillate penicillia. Ten improvements were suggested which appear necessarily complicated.

OTA-producing Penicillium is an illustration of general problems encountered; if such problems are serious here, what then is the situation in less studied taxa? Taxonomic changes in these penicillia have occurred on an average of (less than) every 4 years since 1973. (This does not even include general revisions (e.g., [3]).) A large number of names had previously been given to OTA penicillia, with $P$. verrucosum ultimately being considered as the correct name [15]. The production of OTA, other secondary metabolites, isolation location, colony colour and growth rate were used to delineate "subgroups I to III" within $P$. viridicatum. Thus, $P$. verrucosum was considered as the only OTA-producing species in Penicillium. The concept of chemotypes was introduced, with II being the only one which could produce citrinin. The current situation is that $P$. nordicum and $P$. verrucosum are the producers [13]. P. nordicum is claimed to produce more OTA than $P$. verrucosum, although a statistical analysis was not performed.

Such reports tend to confuse the situation and encourage scepticism of taxonomic schemes. It would be better if the strains were identified as terverticillate, with a simple analysis undertaken for OTA, becoming more sophisticated if necessary. Ref. [13] mentions that non-producing subgroup B1, as described in their paper, may produce under different conditions. It is recommended here that detection limits be assessed. For example, a very dilute sample of positive producers could be analysed and vice versa; and/or increased HPLC injection volumes for negatives and vice versa are possible. The instability in penicillia can be compared to OTA-producing aspergilli [32].

$P$. verrucosum clustered well and OTA was detected in $16 \%$ of strains [3] in a multidisciplinary study in which such characters were given equal weight. However, $21 \%$ of $P$. expansum, $2 \%$ of $P$. solitum, $22 \%$ of Penicillium sp., $1 \%$ of $P$. atromentosum, $1 \%$ of $P$. viridicatum, and $20 \%$ of coremial strains were also listed, indicating production was widely distributed. Some $P$. expansum strains produce a metabolite chromatographically similar to OTA so this species can be discounted. Patulin producers are even more difficult taxonomically, as production is so widespread and uneven within taxa [19,23].

Problems continue: OTA-producing penicillia were isolated at a rate of $93 \%$ and $71 \%$ from grain $([5,6]$ respectively). $P$. viridicatum, $P$. chrysogenum, and $P$. cyclopium were identified conventionally and OTA production was quantified. A wide range of penicillia was isolated in [33], including $P$. chrysogenum, using the same identification methods as $[5,6]$. $P$. verrucosum was not identified. $P$. verrucosum was not isolated from apple production systems [23]. However, P. chrysogenum was identified. There were no OTA fungi found in a cheese factory [34]. The identifications in $[23,33,34]$ were undertaken by experienced taxonomists. The point here is that $P$. chrysogenum was identified in different studies and was demonstrated to be OTA-producing in two and assumed not to be in the others. This indicates that problems can arise from using ambiguous taxonomies. Analysing cultures for particular mycotoxins is recommended irrespective of identifications: It is important in determining weak spots in commodity systems.

The relationship between OTA, ergosterol, and colonyforming units was not direct, except perhaps during logarithmic growth for $P$. verrucosum [31]. Such investigations are valuable (and uncommon) in predicting the situation in vivo. The duration of the experiment could usefully have been extended to determine when OTA reached a steady state or zero, and the corresponding quantity of fungus. The proce- 
dures are recommended over those chosen for preservation of mycotoxin production [29], although liquid culture may be more practicable. The identity of chemicals detected in [28] are unknown (e.g., it is not explained why they were considered as secondary metabolites), and so are not discussed here.

$P$. brevicompactum was isolated at high frequency from (a) grapes $[2,33]$; (b) apple production systems (approximately $40 \%$ had the potential for patulin production) [23]; (c) a cheese production factory (patulin is degraded by proteins in milk) [34]; (d) a water bottling factory (unpublished data); (e) black and white pepper, but not brazil nuts [9]; and (f) cashews [8]. None was identified from grain [5,6]. The associated metabolite deacetylpebrolide was detected in [10]. Fungal metabolites have been recommended for the identifications of fungi in commodities [22]. Given that P. brevicompactum produces patulin and is ubiquitous, it should be considered as a threat to commodity systems.

Interestingly, Peterson [26] sequenced DNA from P. brevicompactum and other isolates with similar conidiogenous structure (Fig. 4). Three clades were revealed which may yield interesting results if analysed for the IDH gene and patulin production [23]. One of the $P$. biourgeiannum strains was received as $P$. patulum; a patulin producer and the synonym of $P$. urticae; the name used for the fungus from which the IDH gene was sequenced. The correct name is $P$. griseofulvum (syn. P. urticae; P. patulum) —other examples of taxonomic complications. P. brevicompactum isozymes (particularly pectinases) were distinctive in [20], and the method may be useful for strains in [26].

Preservation methods are significant in terms of taxonomic studies of fungi. They might kill or alter the specimen. Mycotoxins and secondary metabolites were detected in dead, dried herbarium specimens which had been preserved for decades [21]. This form of preservation could be used to avoid variation in living cultures. Ryan et al. [28] implies that citrinin was "not produced" because of preservation. However, other factors may have been involved: the same analytical procedures were not used before preservation as after, which would have been a more valid protocol. Single strain variation was important in the preservation of citrinin and patulin production and requires systematic investigation [29]. Paterson [19] mentioned that preservation may affect the genome which could distort interpretations involving evolution [35]. The serious issue of inaccurate annotations in public culture collections has been addressed by Montiel et al. [14]. Finally, secondary metabolite production may affect (e.g., inhibit) nucleic acid analysis with implications for taxonomy.

Chemical diversity is very high in fungal extracts; virtually all chemical structures are possible [16]. But this knowledge may be unnecessary for taxonomists or those interested in mycotoxins per se. Name changes are implied in this report, such as $P$. ochrochloron to $P$. simplicissimum, and $P$. crateriforme to $P$. rubrum, which may create further confusion.

\section{A solution}

A more relevant and applicable taxonomy, and preservation procedures are required for the subgenus Penicillium because (a) it is monophyletic, (b) a taxon should reliably produce a particular mycotoxin, and (c) the identification schemes are subjective. Proficiency testing between laboratories is required to assess sampling and identifications. Variation in analyses between strains requires thorough investigation and suitable controls need to be rigorously employed. Finally, any further work is required to be multidisciplinary and encompass taxonomy, mycology, analytical chemistry, molecular biology, image analysis, and bioinformatics.

The primary objective of future work is to ensure that identifications of the subgenus Penicillium indicate unambiguously which of the strains produced the most important mycotoxins of that taxon. A user-friendly yet objective taxonomy is required. It will provide more relevant information than existing schemes.

Techniques are required which are within the operating limits of the method so as to provide reliable information on fungi. Thus, the minutia of fungal morphology can be ignored. The only point to determine is whether a strain has the terverticillate conidiophore structure. Similarly, the details of biochemical techniques can be overlooked in favour of a broad assessment. A non-specialist should be able to readily make these decisions. Only the most important mycotoxins should be considered. Other techniques should only be employed to build on the framework thus obtained. Another innovative concept involves performing the analyses from a single culture of the strains, with advantages gained in terms of subculture variation, contamination, mislabelling, economy, and time. The use of "one agar for all purposes" could be employed instead of "one agar for each test".

Some strains do not produce many secondary metabolites and these could be subjected to simple analysis for universal ubiquinones [18]. Additional novelty could arise from wider use of image analysis techniques to digitise culture morphology and biochemical data. The groups obtained by the morphology and simple TLC and PCR techniques could be analysed further, for example, by RFLP/AFLP methods to determine homogeneity within the groups, although the effects of secondary metabolites on the PCR requires consideration. Sequencing could be applied. Other probes may be used for other relevant metabolites.

Methods of preservation for the continued production of mycotoxins need to be investigated (e.g., subculturing, water storage, silica gel, deep-freezing and freeze-drying [29]). A collection of reference dried cultures and extracts of cultures could usefully be made which contain the relevant mycotoxin(s). The effect of preservation on the genome requires investigation. Reference DNA extracts of metabolic pathways need to be maintained (e.g., the IDH gene). DNA extracts of taxa could be preserved. A useful secondary objective would be to record other metabolites from strains. 
Toward these ends, a culture collection has been established at the Micoteca da Universidade do Minho (MUM), Portugal, dedicated to valid taxonomic research using the most appropriate methods applicable to a wide variety of laboratories [30]. A large biodiversity of fungi has been isolated in studies undertaken by MUM relating to commodity spoilage and mycotoxin contamination. These fungi will form the basis for future taxonomic work.

\section{Conclusions}

The taxonomy of the terverticillate penicillia is unstable, subjective and provides ambiguous information about mycotoxin production. Techniques must fall within the range of most laboratories, without the need for highly trained specialists or expensive equipment, at least in the first phase. However, adopting these procedures means that more useful information will be obtained. A novel approach consists of extracting as much useful taxonomic information as possible from each method within a reasonable time frame, without indulging in excessive detail. Instead of "Penicillium verrucosum", a "subgenus Penicillium-OTA detected" could be obtained.

Added in proof: P. Marek, A. Thirunavukkarasu, K. Venkitanarayanan, Detection of Penicillium expansum by polymerase chain reaction. Int. J. Food Microbiol. 89 (2003) 139-144 is interesting; however, data are required on more strains/taxa (see $[19,20])$.

\section{Acknowledgements}

R.R.M. Paterson was supported by grant SFRH/BPD/ 14923/2004 from the Fundação para a Ciência e a Tecnologia (FCT), Portugal.

\section{References}

[1] M.L. Abarca, M.R. Bragulat, G. Castellá, F.J. Cabañes, Ochratoxin A production by strains of Aspergillus niger var. niger, Appl. Environ. Microbiol. 60 (1994) 2650-2652.

[2] L. Abrunhosa, R.R.M. Paterson, Z. Kozakiewicz, N. Lima, A. Venâncio, Mycotoxin production from fungi isolated from grapes, Let. Appl. Microbiol. 32 (2001) 240-242.

[3] P.D. Bridge, D.L. Hawksworth, Z. Kozakiewicz, A.H.S. Onions, R.R.M. Paterson, M.J. Sackin, P.H.A. Sneath, A reappraisal of the terverticillate penicillia using biochemical, physiological, and morphological features. I. Numerical taxonomy, J. Gen. Microbiol. 135 (1989) 2941-2966.

[4] R. Coker, Mycotoxins-The Silent Threat to Human and Animal Health, in: Inaugural Lecture Series, Procurement and Business Services Department, University of Greenwich, London, 1999, p. 52.

[5] L. Czerwiecki, D. Czajkowska, A. Witkowska-Gwiazdiwska, On ochratoxin A and fungal flora in Polish cereals from conventional and ecological farms. Part 1: The occurrence of ochratoxin A and fungi in cereals in 1997, Food Addit. Contamin. 19 (2002) 470-477.
[6] L. Czerwiecki, D. Czajkowska, A. Witkowska-Gwiazdiwska, On ochratoxin A and fungal flora in Polish cereals from conventional and ecological farms. Part 2: Occurrence of ochratoxin A and fungi in cereals in 1998, Food. Addit. Contamin. 19 (2002) 1051-1057.

[7] T. Dörge, J.M. Carstensen, J.C. Frisvad, Direct identification of pure Penicillium species using image analysis, J. Microbiol. Methods. 41 (2000) 121-133.

[8] F.C.O. Friere, Z. Kozakiewicz, R.R.M. Paterson, Preliminary study of mycoflora and mycotoxins from Brazilian cashews, Mycopathology 145 (1999) 95-103.

[9] F.C.O. Friere, Z. Kozakiewicz, R.R.M. Paterson, Mycoflora and mycotoxins in Brazilian black pepper, white pepper and Brazil nuts, Mycopathology 149 (2000) 13-19.

[10] J.C. Frisvad, O. Filtenborg, F. Lund, R.A. Samson, in: R.A. Samson, J.I. Pitt (Eds.), Integration of Modern Taxonomic Methods for Penicillium and Aspergillus Classification, Harwood Academic, The Netherlands, 2000, pp. 265-283.

[11] J.C. Frisvad, O. Filtenborg, U. Thrane, R.A. Samson, K. Seifert, in: R.A. Samson, J.I. Pitt (Eds.), Integration of Modern Taxonomic Methods for Penicillium and Aspergillus Classification, Harwood Academic, The Netherlands, 2000, pp. 113-125.

[12] S. Kazi, R.R.M. Paterson, N.F. Abo-Dahab, Effect of 2-deoxy-Dglucose on mycotoxins from apples inoculated with Penicillium expansum, Mycopathology 138 (1997) 43-46.

[13] O.L. Larsen, A. Svendsen, J. Smedsgaard, Biochemical characterization of ochratoxin A-producing strains of the genus Penicillium, Appl. Environ. Microbiol 67 (2001) 3630-3635.

[14] D. Montiel, M.J. Dickinson, H.A. Lee, P.S. Dyer, D.J. Jeenes, I.N. Roberts, S. James, L.J. Fuller, K. Matsuchima, D.B. Archer, Genetic differentiation of the Aspergillus section Flavi complex using AFLP fingerprints, Mycol. Res. 107 (2003) 1427-1434.

[15] M.O. Moss, Recent studies of mycotoxins, J. Appl. Microbiol. Symp. Suppl. 84 (1998) 62S-76S.

[16] K.F. Nielsen, J. Smedsgaard, Fungal metabolite screening: Database of 474 mycotoxins and fungal metabolites for dereplication by standardized liquid chromatography-UV-mass spectrometry methodology, J. Chromatog. A 1002 (2003) 111-136.

[17] A.H.S. Onions, P. Bridge, R.R.M. Paterson, Problems and prospects for the taxonomy of Penicillium, Microbiol. Sci. 1 (1984) 185-189.

[18] R.R.M. Paterson, Effect of growth on taxonomically-useful ubiquinone/lipid profiles from Penicillium, Mycol. Res. 97 (1993) 173-178.

[19] R.R.M. Paterson, in: N. Lima, D. Smith (Eds.), Biological Resource Centres and the Use of Microbes, Micoteca da Universidade do Minho, Braga, 2003, pp. 259-266.

[20] R.R.M. Paterson, P.D. Bridge, M.J. Crosswaite, D.L. Hawksworth, A reappraisal of the terverticillate penicillia using biochemical, physiological, and morphological features. III. An evaluation of pectinases and amylase isozymes for species characterization, J. Gen. Microbiol. 135 (1989) 2979-2991.

[21] R.R.M. Paterson, D.L. Hawksworth, Detection of secondary metabolites in dried cultures of Penicillium preserved in herbaria, Trans. Brit. Mycol. Soc. 85 (1985) 95-100.

[22] R.R.M. Paterson, Z. Kozakiewicz, Penicillium and Aspergillus mycotoxins-diagnostic characters and quantitative data from commodities and cultures, Cer. Res. Commun. 25 (1997) 271-275.

[23] R.R.M. Paterson, Z. Kozakiewicz, T. Locke, D. Brayford, S.C.B. Jones, Novel use of the isoepoxydon dehydrogenase gene probe of the patulin metabolic pathway and chromatography to test penicillia isolated from apple production systems for the potential to contaminate apple juice with patulin, Food Microbiol. 20 (2003) 359-364.

[24] R.R.M. Paterson, A. Venâncio, N. Lima, in: N. Lima, D. Smith (Eds.), Biological Resource Centres and the Use of Microbes, Micoteca da Universidade do Minho, Braga, 2003, pp. 217-233.

[25] S.W. Peterson, in: R.A. Samson, J.I. Pitt (Eds.), Integration of Modern Taxonomic Methods for Penicillium and Aspergillus Classification, Harwood Academic, The Netherlands, 2000, pp. 163-178. 
[26] S.W. Peterson, Multilocus DNA sequence analysis shows that Penicillium biourgeianum is a distinct species closely related to P. brevicompactum and P. olsonii, Mycol. Res. 108 (2004), in press.

[27] N. Ratola, L. Martins, A. Alves, Ochratoxin A in wines-assessing global uncertainty associated with the results, Anal. Chim. Acta (2004), in press.

[28] M.J. Ryan, D. Smith, P.D. Bridge, P. Jeffries, The relationship between preservation method and secondary metabolite production in Metarhizium anisopliae and Fusarium oxysporum, World J. Microbiol. Biotechnol. 19 (2003) 839-844.

[29] I.M. Santos, L. Abrunhosa, A. Venâncio, N. Lima, The effect of culture preservation techniques on patulin and citrinin production by Penicillium expansum Link, Let. Appl. Microbiol. 35 (2002) 272-275.

[30] I.M. Santos, N. Lima, Criteria followed in the establishment of a filamentous fungal culture collection-Micoteca da Universidade do Minho (MUM), World J. Microbiol. Biotechnol. 17 (2001) 215-220.
[31] J. Saxena, C. Munimbazi, L.B. Bullerman, Relationship of mould count, ergosterol and ochratoxin A production, Int. J. Food Microbiol. 71 (2001) 29-34.

[32] E. Schuster, N. Dunn-Coleman, J.C. Frisvad, P.W.M. van Dijck, On the safety of Aspergillus niger-a review, App. Microbiol. Biotech. 59 (2002) 426-435.

[33] R. Serra, L. Abrunhosa, Z. Kozakiewicz, A. Venâncio, Black Aspergillus species as ochratoxin A producers in Portuguese wine grapes, Int. J. Food Microbiol. 88 (2003) 63-68.

[34] R. Serra, L. Abrunhosa, Z. Kozakiewicz, A. Venâncio, N. Lima, Use of ozone to reduce molds in a cheese ripening room, J. Food Protect. 66 (2003) 2355-2358.

[35] J. Varga, K. Rigó, J. Molnár, B. Tóth, A. Szencz, J. Téren, Z. Kozakiewicz, Mycotoxin production and evolutionary relationships among species of Aspergillus section Clavati, Anton. v. Leewen. 83 (2003) 191-200. 